\title{
Myasthenic syndrome AChR $\alpha$ C-loop mutant disrupts initiation of channel gating
}

\author{
Xin-Ming Shen, ${ }^{1}$ Joan M. Brengman, ${ }^{1}$ Steven M. Sine, ${ }^{2}$ and Andrew G. Engel ${ }^{1}$ \\ ${ }^{1}$ Neuromuscular Research Laboratory, Department of Neurology, and ${ }^{2}$ Receptor Biology Laboratory, Department of Physiology and Biomedical Engineering, \\ and Department of Neurology, Mayo Clinic, Rochester, Minnesota, USA.
}

\begin{abstract}
Congenital myasthenic syndromes (CMSs) are neuromuscular disorders that can be caused by defects in acetylcholine receptor (AChR) function. Disease-associated point mutants can reveal the unsuspected functional significance of mutated residues. We identified two pathogenic mutations in the extracellular domain of the AChR $\alpha$ subunit $(\mathrm{AChR} \alpha)$ in a patient with myasthenic symptoms since birth: a V188M mutation in the C-loop and a heteroallelic G74C mutation in the main immunogenic region. The G74C mutation markedly reduced surface AChR expression in cultured cells, whereas the V188M mutant was expressed robustly but had severely impaired kinetics. Single-channel patch-clamp analysis indicated that V188M markedly decreased the apparent AChR channel opening rate and gating efficiency. Mutant cycle analysis of energetic coupling among conserved residues within or dispersed around the $\mathrm{AChR} \alpha \mathrm{C}$-loop revealed that $\mathrm{V} 188$ is functionally linked to Y190 in the C-loop and to D200 in $\beta$-strand 10, which connects to the M1 transmembrane domain. Furthermore, V188M weakens inter-residue coupling of K145 in $\beta$-strand 7 with Y190 and with D200. Cumulatively, these results indicate that $\mathrm{V} 188$ of $\mathrm{AChR} \alpha$ is part of an interdependent tetrad that contributes to rearrangement of the $\mathrm{C}$-loop during the initial coupling of agonist binding to channel gating.
\end{abstract}

\section{Introduction}

Congenital myasthenic syndromes (CMSs) are heterogeneous disorders in which the safety margin of neuromuscular transmission is impaired by one or more defects in motor endplate-associated proteins (1). Most defects arise from mutations that occur in subunits of the acetylcholine receptor (AChR) (reviewed in ref. 2); other deleterious mutations have been identified in choline acetyltransferase (ChAT) (3); the collagenic tail subunit of the endplate species of acetylcholinesterase (AChE) $(4,5)$; the cytoplasmic protein rapsyn (6); agrin (7); the muscle-specific receptor tyrosine kinase (MuSK) (8); Dok-7, a muscle-intrinsic activator of MuSK (9); plectin (10); glutamine-fructose-6-phosphate transaminase 1 (GFPT1) (11); and the $\mathrm{Na}_{v} 1.4$ sodium channel (12). The pathogenic mutations in AChR subunits either decrease the expression or alter the kinetics of activation of the receptor by nerve-released ACh. The mutations that alter activation kinetics result in physiologically opposite slow- and fast-channel syndromes that prolong or speed the decay of the synaptic current (13).

The AChR is a ligand-gated ion channel in which 5 homologous subunits align side by side to form a cylindrical structure lodged within the cell membrane. At the innervated adult endplate (EP) the stoichiometry of the subunits is $(\alpha 1)_{2} \beta 1 \delta \varepsilon$, whereas at denervated or developing EPs the fetal $\gamma$ subunit is present in place of the $\varepsilon$ subunit. Each subunit includes a large extracellular domain, 4 transmembrane domains, and a large intracellular domain (14). The extracellular domain consists of an inner core of $6 \beta$-strands and an outer shell of $4 \beta$-strands, whereas the transmembrane domains are $\alpha$-helices (15). The 2 agonist-binding pockets of $\mathrm{AChR}$ are located at interfaces between the $\alpha$ subunits and the neighboring $\varepsilon$ or $\delta$ subunits. The principal face of each binding site is formed by the $\alpha$ subunit, which contributes peptide loops A-C, while the complimentary face is formed by either the $\delta$ or the

Conflict of interest: The authors have declared that no conflict of interest exists. Citation for this article: J Clin Invest. 2012;122(7):2613-2621. doi:10.1172/JCI63415. $\varepsilon$ subunit contributing loops D-G (16). A structural transitional zone between the ligand-binding and channel domains transduces conformational changes resulting from agonist binding into opening of the ion channel.

Crystallographic studies of isolated binding domains of the mouse AChR (17) and of the ACh binding protein of the freshwater snail and the saltwater mollusk $(18,19)$ as well as cryoelectron microscopy analysis of Torpedo AChR at 4-Å resolution (15) rationalized previous observations on the functional properties of the receptor but did not reveal how different residues or domains of the receptor contribute to the transition from the closed to the open state. Subsequent studies, however, attained this goal by exploring energetic coupling among residues within or between subunits by combined mutagenesis studies, single-channel recordings, and thermodynamic mutant cycle analyses (20-24). These studies revealed that activation of the receptor begins by transition of the C-loop of each $\alpha$ subunit from an opened-up to a closed-in conformation (24-26); this leads to displacement of $\beta$-strand 10 , which is contiguous with the pre-M1 domain, and results in conformational changes of the $\beta 1-\beta 2$ linker and the Cys-loop (Figure 1). Both the $\beta 1-\beta 2$ linker and the Cys-loop energetically couple with residues in the M2-M3 linker and in the course of channel opening displace the M2 helix away from the center of pore $(21,23,27)$.

In this study we identified a p.V188M mutation in the C-loop of the AChR $\alpha 1$ subunit that disrupts the initial coupling of agonist binding to channel gating. Unlike previously identified residues that affect coupling, $\alpha$ Val188 is conserved only in the muscle AChR $\alpha 1$ subunit but not in $\alpha$ subunits of other AChR subtypes. Single-channel recordings from HEK cells expressing the $\alpha V 188 \mathrm{M}$ receptor reveal very brief channel openings flanked by prolonged closings. Estimates of activation rate constants for the $\alpha \mathrm{V} 188 \mathrm{M}$ AChR from single-channel recordings, obtained over a range of ACh concentrations, show that $\alpha \mathrm{V} 188 \mathrm{M}$ profoundly affects the apparent channel opening rate and gating efficiency of the recep- 


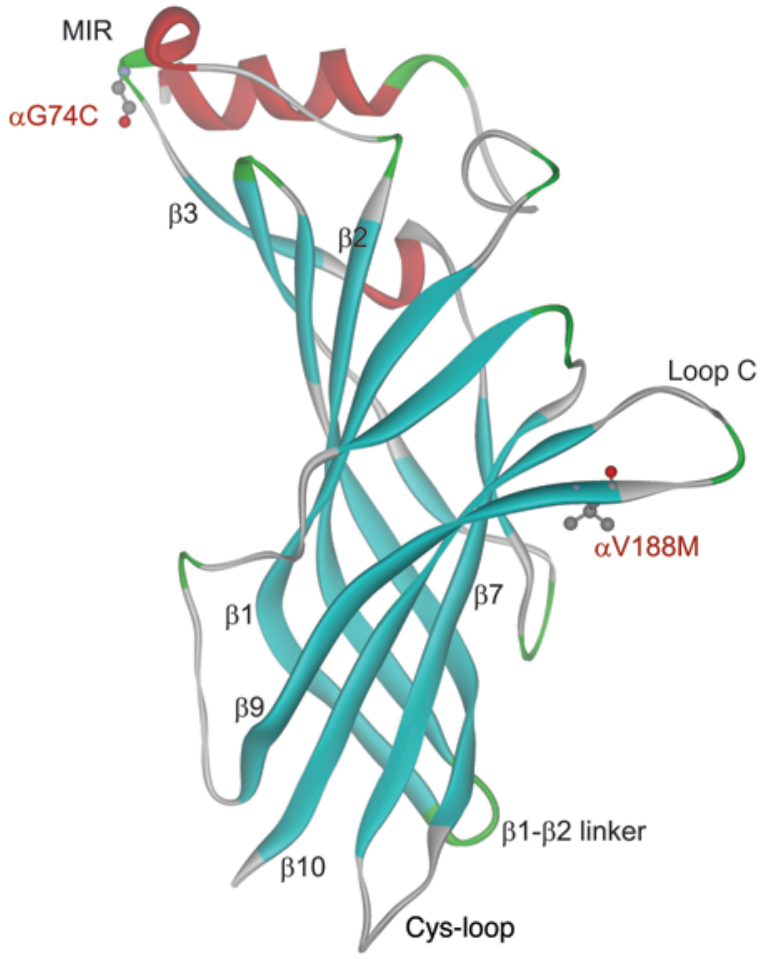

Figure 1

Structural model of the mouse AChR $\alpha$ subunit at $1.94-\AA$ resolution (PDB 2QC1) showing positions of the identified mutations. MIR, main immunogenic region.

tor, effects that are independent of the size of the substituting residue. Finally, using mutant cycle analyses (MCA) we show that $\alpha$ Val188 is part of a network of residues that link agonist-mediated conformational changes in loop $\mathrm{C}$ to subsequent structural rearrangements that culminate in opening the AChR channel.

\section{Results}

Characteristics of the CMS patient. The patient, a woman 42 years old at the time of the study, was born with floppy infant syndrome, with a feeble cry and poor sucking reflex. She had recurrent aspirations and droopy eyelids. She walked at 12 months but often fell. Later she had ocular muscle weakness, abnormal fatigability, and generalized weakness. Thymectomy at age 8 years was of no benefit. At age 14, repetitive stimulation of the femoral nerve at $2 \mathrm{~Hz}$ revealed a $66 \%$ decremental response of the first compared with the fourth evoked compound action potential from the rectus femoris muscle. She had no anti-AChR antibodies. Light and electron microscopy studies of intercostal muscle neuromuscular junctions at age 14 revealed no structural abnormality. The patient's symptoms responded partially to pyridostigmine. There was no history of similarly affected family members.

Mutation analysis. To determine the molecular basis of the patient's illness, we sequenced each AChR subunit gene. This revealed two heterozygous mutations in CHRNA1: p. $\alpha \mathrm{G} 74 \mathrm{C}$ (c.280G>T) and p. $\alpha$ V188M (c.622G>A). (Nucleotide numbers start from the translational start site, with +1 corresponding to the A of the ATG translation initiation codon [NM_000079.3]; codon numbers start from the first codon of the mature peptide [NP_000070.1]). $\alpha$ G74C is in the main immunogenic region (MIR) and $\alpha$ V188M in the C-loop of the agonist-binding domain of the $\alpha$ subunit (Figure 1 ). The mutated Gly74 is conserved across the AChR $\alpha 1$ and $\beta$ subunits of different species and in all human AChR $\alpha$ subunits except in $\alpha 2$ and $\alpha 4$. The mutated Val188 is conserved across $\alpha 1$ subunits of different species (Figure 2) but not in $\alpha$ subunits of other AChR subtypes. Neither mutation is present in 400 normal alleles of 200 unrelated subjects. Family analysis indicated that each mutation is recessive, the paternal and maternal alleles harboring $\alpha \mathrm{G} 74 \mathrm{C}$ and $\alpha \mathrm{V} 188 \mathrm{M}$, respectively.

Expression studies in HEK cells. To assess pathogenicity of the observed mutations, we engineered each mutation into the human $\alpha$ subunit and co-transfected cDNAs encoding either mutant or wild-type $\alpha$ subunits with complementary wild-type $\beta, \delta$, and $\varepsilon$ subunits into HEK cells (Figure 3A). To assess expression of the AChR on the cell surface, we measured the binding of $\left[{ }^{125} \mathrm{I}\right] \alpha$-bungarotoxin ([125I] $\alpha$-bgt) to intact cells (Figure 3A). Surface expression of $\alpha$ G74C AChR was reduced to $14 \%$ that of wild-type and that of $\alpha$ V188M AChR was close to normal. Therefore, $\alpha \mathrm{V} 188 \mathrm{M}$ determines the phenotype.

Activation kinetics of the $\alpha V 188 M$ mutant. The safety margin of neuromuscular transmission depends critically on the ability of nerve-released ACh to rapidly and efficiently activate AChR channels. To identify elementary kinetic steps in AChR activation altered by $\alpha V 188 \mathrm{M}$, we first compared the activation of wild-type and mutant receptors expressed in HEK cells by analyzing the single-channel currents elicited by limiting low concentrations of ACh. A concentration of $50 \mathrm{nM}$ ACh elicited a sufficient number of channel openings from wild-type AChR, but mutant AChR required $1 \mu \mathrm{M}$ ACh (Figure $3 \mathrm{~B}$ ). The resulting channel openings

\begin{tabular}{|c|c|c|c|c|c|c|c|}
\hline$\alpha 1$ & \multicolumn{2}{|c|}{$\beta 7$} & $\beta 9$ & Loop C & & \multicolumn{2}{|r|}{$\beta 10$} \\
\hline Human & $\ldots$ QNCSM & $\mathrm{K}$ & LGTWTY . . . GEWVIKE & $\mathrm{V} \mathrm{T}$ & Y SCCPDTPYL & D & $\mathrm{RI}$ \\
\hline impanzee & . QNCSM & $\mathrm{K}$ & LGTWTY . . GEWVIKESRGWKHS & $\mathrm{V} / \mathrm{T}$ & Y SCCPDTPYL & $D$ & RLP \\
\hline Dog & $\ldots$ QNCSM & K & LGTWTY . . . GEWVIKESRGWKHW & $\mathrm{V} \mid \mathrm{F}$ & Y & $D$ & RLP \\
\hline Cattle & $\ldots q$ & K & LGTWTY . . . GEWVIKE & \begin{tabular}{l|l|l}
$\mathrm{V}$ & $\mathrm{F}$
\end{tabular} & Y ACCPSTPYL & $\mathrm{D}$ & RLP \\
\hline Mouse & $\cdots Q$ & $\mathrm{~K}$ & LGTWTY . . . GE & $\mathrm{V} / \mathrm{F}$ & Y SCCPTTPYL & $D$ & $\operatorname{ITY}$ \\
\hline Rat & $\ldots$ QNCSM & $\mathrm{K}$ & LGTWTY . . . GEWVIKEARGWKHW & $\mathrm{V} / \mathrm{F}$ & Y SCCPNTPYL & $D$ & ITY \\
\hline Chicken & $\ldots$ QNCSM & $\mathrm{K}$ & LGTWTY . . . GEWVMKDYRGWKHW & $\mathrm{V}$ & Y ACCPDTPYL & $D$ & ITY \\
\hline Zebrafish & $\ldots$ QNCSM & $\mathrm{K}$ & LGTWTY . . . GEWVMKDYRSWKHW & $\mathrm{V} / \mathrm{Y}$ & Y ACCPDTPYL & $D$ & ITYHFLLLRLP \\
\hline & 2019 & T & LGTWTY . . . GEWVIKESRGWKHS & $\mathrm{M} \mathrm{T}$ & Y SCCPDTPYL & $D$ & ITYHFVMQRLP \\
\hline & & & & & & (2) & \\
\hline
\end{tabular}

Figure 2

Sequence alignment of the $\alpha 1 \mathrm{AChR}$ subunits in different species. 
A
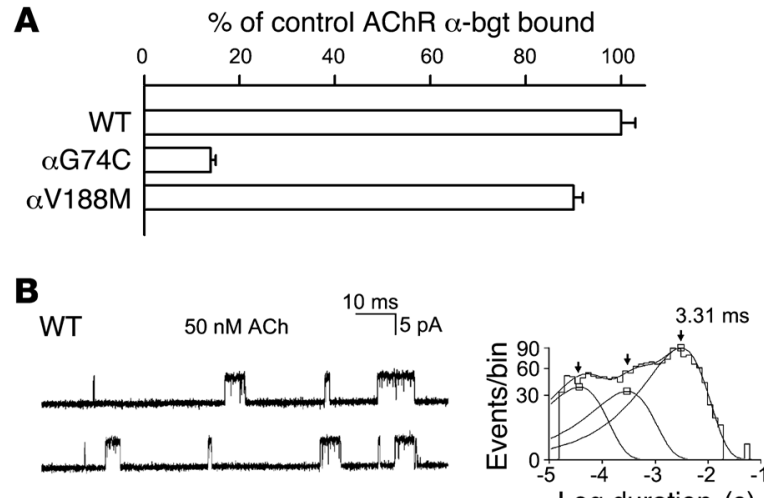

Log duration (s)

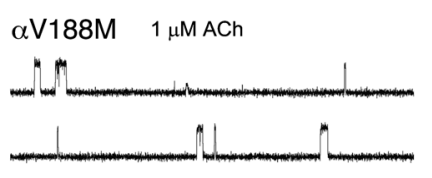

\section{Figure 3}

$\alpha$-bgt binding studies and single-channel currents elicited by ACh. (A) $\left[{ }^{125}\right] \alpha$-bgt binding to surface receptors on intact HEK cells transfected with wild-type and mutant AChRs. The results are normalized for $\alpha$-bgt binding to wild-type AChR and represent mean \pm SD of 3-6 experiments. (B) Single-channel currents elicited by ACh from HEK cells expressing wild-type and mutant AChRs. Left: Representative channel openings shown as upward deflections. Right: Logarithmically binned burst duration histograms fitted to the sum of exponentials. Arrows indicate mean durations of dominant burst components. appeared either as isolated openings or as several openings in quick succession, called bursts, the duration of which mirrors the rate of decay of the postsynaptic current. Construction of histograms of burst dwell times and fitting them with sums of exponentials revealed 2 components for $\alpha \mathrm{V} 188 \mathrm{M}$ and 3 components for wild-type AChR (Figure 3B and Table 1). For the wild-type $\mathrm{AChR}$, the mean duration of the longest and predominant burst component was $3.31 \mathrm{~ms}$, but for the mutant it was only $0.68 \mathrm{~ms}$.

To identify the kinetic steps responsible for the short-lived bursts of the mutant receptor, we recorded single-channel currents over a range of ACh concentrations and constructed histograms of the resulting open and closed dwell times (see Methods). For wild-type AChR, ACh concentrations higher than $10 \mu \mathrm{M}$ elicited well-defined clusters of events, all from the same AChR channel, but for the $\alpha$ V188M AChR the threshold for forming clusters was $30 \mu \mathrm{M}$ ACh (Figure 4). For both wild-type and mutant receptors, the dominant component of closed dwell times shifted to shorter durations with increasing ACh concentrations, but for the mutant receptor the shift toward brief closed durations was much smaller, and at saturating concentrations the closed durations remained prolonged (Figure 4). Because the ACh-dependent shift from long to brief closed durations signals a process driven by binding of ACh, the prolonged closed durations for $\alpha \mathrm{V} 188 \mathrm{M}$ AChR indicate a change in one or more elementary kinetic steps that precede opening of the channel. The open duration histograms changed little across the range of ACh concentrations and remained consistently briefer for the mutant than the wild-type receptor (Figure 4). Thus, $\alpha \mathrm{V} 188 \mathrm{M}$ destabilizes the di-liganded open channel state.

To quantify changes in elementary rate constants underlying activation of the $\alpha V 188 \mathrm{M} A C h R$, we next analyzed the open and closed dwell times according to a linear scheme of receptor activation that recognizes 3 closed and 1 open state of the receptor (Figure 5). In this scheme, 2 agonists (A) bind to the receptor (R) with association rate constants $k_{+1}$ and $k_{+2}$, and dissociate with rate constants $k_{-1}$ and $k_{-2}$. The receptor occupied by 2 agonists opens with rate constant $\beta$ and closes with rate constant $\alpha$. At high concentrations, ACh blocks the open channel with rate $k_{+b}$, and the channel unblocks with rate $k_{-b}$. Owing to bandwidth limitations, this scheme omits the recently described intermediate closed state between $\mathrm{A}_{2} \mathrm{R}$ and $\mathrm{A}_{2} \mathrm{R}$ * (asterisk indicates the open state of the receptor) (28), so the fitted channel gating rate and equilibrium constants presented here are apparent constants.

For both wild-type and mutant $\alpha$ V188M AChRs, probability density functions computed from the fitted rate constants describe the dwell time distributions for the entire range of ACh concentrations. The analysis provides estimates of rate constants for ACh association and dissociation and of apparent rate constants for opening and closing of the channel (Table 2). The most significant effect of $\alpha \mathrm{V} 188 \mathrm{M}$ was an approximately 70 -fold decrease in the channel gating efficiency $\theta$, defined by the ratio of rate constants $\beta / \alpha$, which was largely due to an approximately 80 -fold decrease in $\beta$. The subtle decrease in the closing rate con-

Table 1

Burst durations of wild-type and mutant AChRs in HEK cells

$\begin{array}{lccc} & & \text { Bursts } & \tau_{3} \text {, ms (area) } \\ \text { Wild-type }(n=21) & \tau_{1}, \text { ms (area) } & \tau_{2}, \text { ms (area) } & 3.31 \pm 0.12(0.58 \pm 0.04)^{\mathrm{B}} \\ \alpha \operatorname{V188M~}(n=5) & 0.10 \pm 0.017(0.37 \pm 0.078) & 0.002 \pm 0.06(0.21 \pm 0.03) & \end{array}$

Values indicate mean \pm SEM number of patches. Values in parentheses indicate histogram areas. ACh concentration was 50 nM for wild-type and $1 \mu \mathrm{M}$ for $\alpha \mathrm{V} 188 \mathrm{M} \mathrm{AChR}$. The temperature was $22^{\circ} \mathrm{C} \pm 0.5^{\circ} \mathrm{C}$. The bandwidth was $11.7 \mathrm{kHz}$. The membrane potential was $-80 \mathrm{mV}$. ${ }^{\mathrm{A} N o t}$ detected at 3 patches. ${ }^{\mathrm{B} P}<0.001$, between dominant components of wild-type and mutant burst openings. 
A

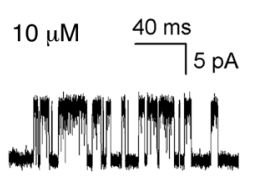

$30 \mu \mathrm{M}$

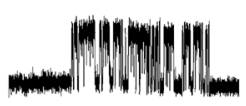

$100 \mu \mathrm{M}$
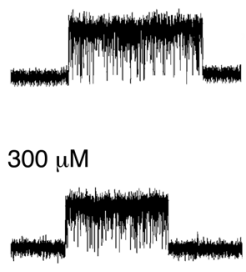

B $\alpha \mathrm{V} 188 \mathrm{M}$

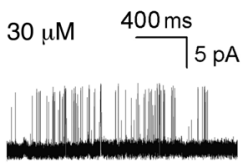

$100 \mu \mathrm{M} \quad 200 \mathrm{~ms}$
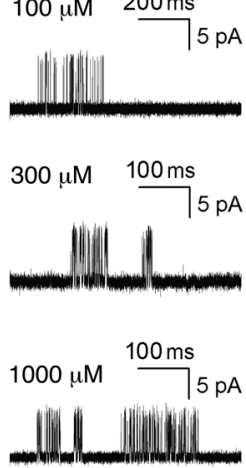

Closed
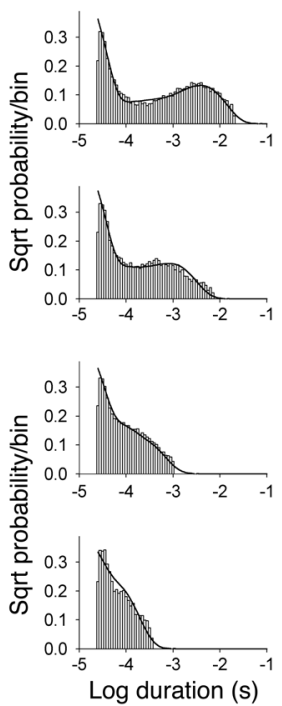

Closed
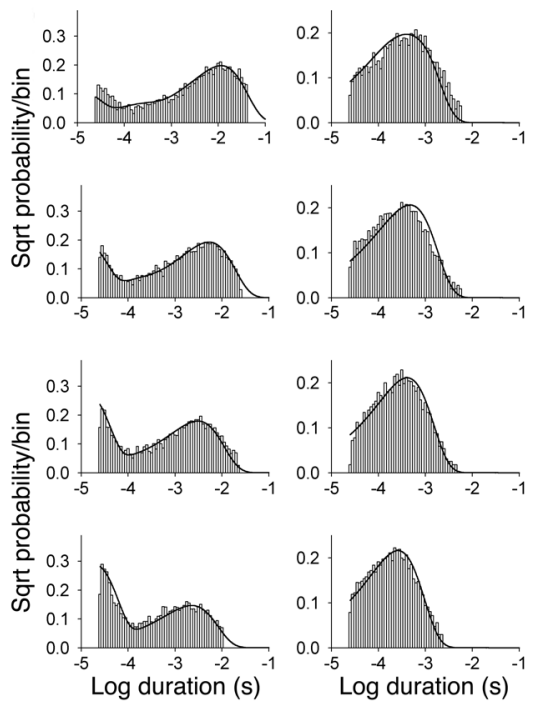

stant $\alpha$ in the mutant receptor, shown in Table 2 , suggests that the briefer mutant receptor open times in Figure 4 derive from fewer missed closings in the mutant than wild-type receptor.

The fitted rate constants in Table 2 allow calculation of the channel open probability $\left(P_{\text {open }}\right)$ as a function of ACh concentration. A plot of $P_{\text {open }}$ over 3 orders of magnitude of ACh concentration revealed a marked decrease and rightward shift of $P_{\text {open }}$ of the mutant relative to the wild-type AChR (Figure 6). The plotted points are well described by the $P_{\text {open }}$ curve computed from the fitted rate constants and support the validity of the estimated rate constants shown in Table 2. The fitted rate constants in Table 2 also allow prediction of the mean burst duration of the $\alpha \mathrm{V} 188 \mathrm{M}$ receptor, approximated by $\left(1+\beta / k_{-2}\right) / \alpha$. The predicted value of $0.55 \mathrm{~ms}$ agrees well with the independently determined burst duration of $0.68 \mathrm{~ms}$ in the presence of $1 \mu \mathrm{M}$ ACh (Table 1). The shortened burst duration predicts an abnormally fast decay of synaptic

\section{Figure 4}

Activation kinetics of wild-type and $\alpha \mathrm{V} 188 \mathrm{M}$ AChR. Left: Representative single-channel currents at the indicated $A C h$ concentrations recorded from HEK cells expressing (A) wild-type and (B) $\alpha \mathrm{V} 188 \mathrm{M}$ AChRs. Currents are shown as upward deflections; bandwidth, $10 \mathrm{kHz}$. Center and right: Histograms of closed and open durations corresponding to each ACh concentration are shown with the probability density functions (smooth curves) generated from a global fit of the scheme to dwell times obtained for the entire range of ACh concentrations. Fitted rate constants are shown in Table 2. On the $y$ axes, each histogram entry is the probability of occurrence on a square root (Sqrt) scale.

currents typical of a fast-channel myasthenic syndrome (13). The probability the channel opens once it has bound two molecules of ACh, obtained from $\beta /\left(\beta+k_{-2}\right)$, was 0.84 for wild-type AChR and 0.11 for $\alpha V 188 \mathrm{M} A C h R$, predicting a reduced peak of the synaptic response to nerve impulse.

In summary, patch-clamp analysis of the kinetic properties of $\alpha$ V188M AChR revealed reduced gating efficiency, abnormally brief channel opening events, a decreased probability of channel opening by ACh, and a decreased amplitude of the synaptic response to ACh. The overall physiological consequence is a markedly reduced safety margin for neuromuscular transmission.

Other substitutions of $\alpha 1 \mathrm{Val}$ at codon 188. Substituting Met for $\mathrm{Val}$ at codon 188 increases side chain size. To determine whether this can account for the adverse effects of the mutation, we replaced $\alpha$ Val188 by Gly, Ala, and Thr, all smaller than Val, and by Leu, which is larger than Val. Each substitution reduced rather than enhanced the apparent channel opening rate constant and gating efficiency, with Gly having the greatest and Thr the smallest effect (Table 2).

Mutant cycle analysis. The available structural data show that the C-loop changes its conformation in the course of agonist binding. Without agonist, the C-loop adopts a range of opened-up conformations that allow agonist access to the binding pocket, but when the agonist binds the C-loop closes inward and traps the agonist $(24,26,29,30)$. Concomitantly, electrostatic interactions among local conserved residues change: without agonist, $\alpha$ Asp200 on $\beta$-strand 10 adjacent to the C-loop and $\alpha$ Lys 145 on $\beta$-strand 7 form a salt bridge, but upon binding the agonist, $\alpha$ Tyr190 on the C-loop approaches $\alpha$ Lys 145, forming a hydrogen bond $(20,24)$ (Figure 7A). Mutation analysis combined with single-channel kinetic analysis revealed that these 3 residues, conserved among muscle and most neuronal AChRs, are energetically coupled in contributing to channel gating, and thus initiate the chain of events that ultimately open the receptor ion channel (20). Because high-resolution structural data showed that $\alpha$ Val188 lodges between $\alpha$ Asp200 and $\alpha$ Tyr190 (Figure 7A), we used mutant cycle analysis (MCA) to determine whether $\alpha$ Val188 is part of the network of residues involved in the initial coupling of agonist binding to channel gating.

A founding principle in MCA is that the change in free energy caused by a mutation depends on other residues in the protein (31). If mutation of a second residue affects the free energy

$$
A+R \underset{k_{-1}}{\stackrel{k_{+1}}{\rightleftarrows}} A R+A \underset{k_{-2}}{\stackrel{k_{+2}}{\rightleftharpoons}} A_{2} R \underset{\alpha}{\stackrel{\beta}{\rightleftharpoons}} A_{2} R^{*} \underset{k_{-b}}{\stackrel{k_{+b}}{\rightleftharpoons}} A_{2} R_{B}
$$

\section{Figure 5}

Scheme of AChR activation. 
Table 2

Kinetic analysis of AChRs with mutations in the $\alpha$ subunit

\begin{tabular}{|c|c|c|c|c|c|c|c|c|c|c|c|c|c|}
\hline & $k_{+1}$ & $k_{-1}$ & $\begin{array}{c}K_{1} \\
(\mu M)\end{array}$ & $k_{+2}$ & $k_{-2}$ & $\begin{array}{c}K_{2} \\
(\mu \mathrm{M})\end{array}$ & $\beta$ & $\alpha$ & $\theta$ & $k_{+b}$ & $k_{-b}$ & $\begin{array}{c}K_{B} \\
(\mu \mathrm{M})\end{array}$ & $\begin{array}{c}\Delta G^{o} \\
(\mathrm{kcal} / \mathrm{mol})\end{array}$ \\
\hline Wild-type & $\begin{array}{l}81 \\
\pm 6\end{array}$ & $\begin{array}{l}1,036 \\
\pm 108\end{array}$ & 13 & $\begin{array}{l}84 \\
\pm 2\end{array}$ & $\begin{array}{c}10,679 \\
\pm 167\end{array}$ & 127 & $\begin{array}{r}57,139 \\
\pm 1,219\end{array}$ & $\begin{array}{l}2,410 \\
\pm 65\end{array}$ & 24 & $\begin{array}{l}27 \\
\pm 2\end{array}$ & $\begin{array}{l}103,239 \\
\pm 2,480\end{array}$ & 3.82 & \\
\hline V188M & ND & ND & - & $\begin{array}{l}22 \\
\pm 1\end{array}$ & $\begin{array}{l}6,034 \\
\pm 478\end{array}$ & 270 & $\begin{array}{l}730 \\
\pm 14\end{array}$ & $\begin{array}{l}2046 \\
\pm 15\end{array}$ & 0.35 & $\begin{array}{l}34 \\
\pm 1\end{array}$ & $\begin{array}{r}106,423 \\
\pm 1,645\end{array}$ & 3.13 & 2.48 \\
\hline V188G & ND & ND & - & $\begin{array}{l}39 \\
\pm 6\end{array}$ & $\begin{array}{l}5,634 \\
\pm 921\end{array}$ & 144 & $\begin{array}{l}330 \\
\pm 6\end{array}$ & $\begin{array}{c}1,102 \\
\pm 10\end{array}$ & 0.30 & $\begin{array}{l}17 \\
\pm 1\end{array}$ & $\begin{array}{r}67,713 \\
\pm 1,566\end{array}$ & 3.98 & 2.57 \\
\hline V188A & ND & ND & - & $\begin{array}{l}53 \\
\pm 1\end{array}$ & $\begin{array}{c}12,275 \\
\pm 373\end{array}$ & 231 & $\begin{array}{c}2,616 \\
\pm 44\end{array}$ & $\begin{array}{c}1,420 \\
\pm 10\end{array}$ & 1.84 & $\begin{array}{l}29 \\
\pm 2\end{array}$ & $\begin{array}{l}121,761 \\
\pm 2,601\end{array}$ & 4.20 & 1.51 \\
\hline V188T & $\begin{array}{l}64 \\
\pm 7\end{array}$ & $\begin{array}{l}4,376 \\
\pm 764\end{array}$ & 68 & $\begin{array}{l}174 \\
\pm 46\end{array}$ & $\begin{array}{r}50,839 \\
\pm 8,763\end{array}$ & 292 & $\begin{array}{l}8,746 \\
\pm 634\end{array}$ & $\begin{array}{l}1,104 \\
\pm 25\end{array}$ & 7.92 & $\begin{array}{l}18 \\
\pm 1\end{array}$ & $\begin{array}{c}88,662 \\
\pm 2,788\end{array}$ & 4.92 & 0.65 \\
\hline V188L & ND & ND & - & $\begin{array}{l}42 \\
\pm 1\end{array}$ & $\begin{array}{l}8,963 \\
\pm 456\end{array}$ & 213 & $\begin{array}{l}4,818 \\
\pm 125\end{array}$ & $\begin{array}{l}1,078 \\
\pm 12\end{array}$ & 4.47 & $\begin{array}{l}21 \\
\pm 1\end{array}$ & $\begin{array}{r}97,056 \\
\pm 1,775\end{array}$ & 4.62 & 0.99 \\
\hline Y190F & ND & ND & - & ND & ND & - & $\begin{array}{l}38 \\
\pm 2\end{array}$ & $\begin{array}{l}600 \\
\pm 27\end{array}$ & 0.063 & $\begin{array}{l}18 \\
\pm 2\end{array}$ & $\begin{array}{r}60,789 \\
\pm 4,140\end{array}$ & 3.37 & 3.48 \\
\hline K145E & $\begin{array}{c}34 \\
\pm 10\end{array}$ & $\begin{array}{l}1,377 \\
\pm 207\end{array}$ & 41 & $\begin{array}{c}64 \\
\pm 11\end{array}$ & $\begin{array}{r}14,936 \\
\pm 2,169\end{array}$ & 233 & $\begin{array}{l}648 \\
\pm 35\end{array}$ & $\begin{array}{l}1,035 \\
\pm 15\end{array}$ & 0.63 & $\begin{array}{l}18 \\
\pm 1\end{array}$ & $\begin{array}{r}74,299 \\
\pm 3,464\end{array}$ & 4.13 & 2.13 \\
\hline $\mathrm{D} 200 \mathrm{H}$ & $\begin{array}{c}6 \\
\pm 1\end{array}$ & $\begin{array}{r}134 \\
\pm 17\end{array}$ & 22 & $\begin{array}{l}31 \\
\pm 3\end{array}$ & $\begin{array}{l}6,068 \\
\pm 567\end{array}$ & 195 & $\begin{array}{l}582 \\
\pm 23\end{array}$ & $\begin{array}{l}1,282 \\
\pm 13\end{array}$ & 0.45 & $\begin{array}{l}12 \\
\pm 1\end{array}$ & $\begin{array}{l}72,352 \\
\pm 2,570\end{array}$ & 6.02 & 2.33 \\
\hline Y93F & ND & ND & - & $\begin{array}{l}20 \\
\pm 1\end{array}$ & $\begin{array}{r}10,675 \\
\pm 1,066\end{array}$ & 533 & $\begin{array}{l}1,167 \\
\pm 40\end{array}$ & $\begin{array}{l}1,583 \\
\pm 15\end{array}$ & 0.74 & $\begin{array}{l}21 \\
\pm 1\end{array}$ & $\begin{array}{l}107,998 \\
\pm 2,374\end{array}$ & 5.14 & 2.04 \\
\hline
\end{tabular}

Kinetic parameters and error estimates are derived from global fitting of a kinetic scheme to data obtained over a wide range of $A C h$ concentrations. Rate constants are $\mu \mathrm{M}^{-1} \times \mathrm{s}^{-1}$ for association rate constants and $\mathrm{s}^{-1}$ for all others. The dissociation constants, $K_{1}$ and $K_{2}$, are the ratios of $k_{-}$to $k_{+}$. $\Delta G^{\circ}$ indicates free energy change in $\mathrm{kcal} / \mathrm{mol}$. The channel gating equilibrium constant $\theta$ is the ratio of the opening rate $(\beta)$ to closing rate $(\alpha)$ constants. The blocking rate equilibrium constant $K_{B}$ equals the unblocking rate $k_{-b}$ divided by the blocking rate $k_{+b}$. Free energy change: $\Delta G{ }^{\circ}=-R T$ In $(\theta$ mutant $/ \theta$ wild-type), where $R$ is the gas constant $\left(1.987 \mathrm{cal} /{ }^{\circ} \mathrm{K} / \mathrm{mol}\right)$ and $T$ is the absolute temperature $\left(295^{\circ} \mathrm{K}\right)$. ND, reaction step not detected.

change caused by mutation of the first, the two residues are energetically coupled and potentially interact directly. Evidence for direct interaction depends on independent structural data showing physical proximity, which for the $\alpha 1$ extracellular domain show that $\alpha$ Val188 is adjacent to several conserved residues essential for gating (17). For the present application of MCA, the free energy change caused by each mutation is computed from the channel gating equilibrium constant $\theta$ derived from kinetic fitting of single-channel dwell times, which equals $-R T \ln \theta$, where $R$ is the gas constant and $T$ absolute temperature. To compute energetic coupling between 2 residues, free energy changes were computed for wild-type, each single mutant, and the double mutant AChR, and the differences in free energy between pairs of residues are shown along 4 lines that form a square. If the free energy changes along parallel lines of the square are the same, the 2 residues are not coupled, whereas if they differ, they are coupled. For compact illustration, the present MCA data are cast as cubes, with each face of the cube representing the free energy of coupling between pairs of residues.

We first tested for energetic coupling between $\alpha$ Val188 and either $\alpha$ Tyr 190 or $\alpha$ Asp 200 . To this end, we generated mutations of each residue individually and of pairs of residues in the same

\section{Figure 6}

$P_{\text {open }}$ as a function of ACh concentration. Symbols and vertical lines indicate mean \pm SD and represent $P_{\text {open }}$ computed from 29 to 300 clusters in a single patch. Smooth curves indicate the $P_{\text {open }}$ predicted by the fitted rate constants shown in Table 2.

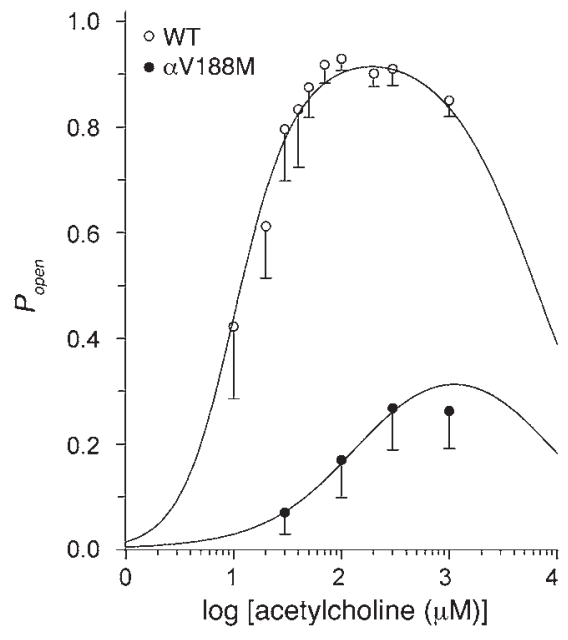


Table 3

Inter-residue energetic coupling determined by MCA

\begin{tabular}{lcccc} 
& $\beta\left(\mathbf{s}^{-1}\right)$ & $\alpha\left(\mathbf{s}^{-1}\right)$ & $\theta(\beta / \alpha)$ & $\Delta \Delta \boldsymbol{G}_{\text {int }}(\mathbf{k c a l} / \mathbf{m o l})$ \\
V188M-Y190F & $62 \pm 2$ & $991 \pm 30$ & 0.062 & -2.47 \\
K145E-V188M & $38 \pm 2$ & $1,096 \pm 40$ & 0.035 & -0.78 \\
D200H-V188M & $692 \pm 32$ & $1,393 \pm 86$ & 0.49 & -2.53 \\
K145E-Y190F & $29 \pm 1$ & $1,522 \pm 50$ & 0.019 & -1.43 \\
K145E-D200H & $300 \pm 21$ & $1,505 \pm 35$ & 0.20 & -1.66 \\
Y93F-V188M & $21 \pm 0.4$ & $2,105 \pm 43$ & 0.010 & 0.04 \\
K145E-V188M-Y190F & $35 \pm 1$ & $2,375 \pm 50$ & 0.015 & \\
K145E-V188M-D200H & $127 \pm 5$ & $2,191 \pm 80$ & 0.058 & \\
\hline
\end{tabular}

Rate constants and error estimates are derived from global fitting of a kinetic scheme to data obtained over a wide range of ACh concentrations. Apparent gating equilibrium constants $(\theta)$ are the ratio of the channel opening $(\beta)$ to closing rate constants $(\alpha)$. For 2-dimensional mutant cycles, first-order coupling free energies are represented by $\Delta \Delta G_{\text {int }}$.

getic coupling in this residue pair, shown by the front faces of the cubes in Figure 7, B and C, was low, with a $\Delta \Delta G_{\text {int }}$ of only $-0.78 \mathrm{kcal} / \mathrm{mol}$ (Table 3 ). Although $\alpha$ Val188 and $\alpha$ Lys 145 were weakly coupled, we found that the coupling free energy was affected by mutations of either $\alpha$ Tyr 190 or $\alpha$ Asp200. Comparison of the front and back faces (Figure 7B) shows that mutation of $\alpha$ Tyr 190 reduces coupling from -0.78 to 0.13 $\mathrm{kcal} / \mathrm{mol}$, while comparison of the front and back faces (Figure 7C) shows that mutation of $\alpha$ Asp200 altered coupling from -0.78 to $0.78 \mathrm{kcal} / \mathrm{mol}$. Our collective findings show that residue pairs that exhibit strong energetic coupling make direct contact, whereas those that couple weakly do not. Moreover, $\alpha$ Val188 joins $\alpha$ Tyr190, $\alpha$ Asp200, and $\alpha$ Lys 145 to form an interdependent tetrad that links agonistmediated changes in the C-loop to channel gating.

A recently reported crystal structure of the ago-

subunit, recorded single-channel currents for each mutant AChR, computed the apparent channel gating equilibrium constant that yielded gating free energy, and constructed 2-dimensional mutant cycles shown as faces of a 3-dimensional cubic cycle (Figures 7, $\mathrm{B}$ and $\mathrm{C}$ ). The analyses indicate that $\alpha$ Val188 couples strongly to both $\alpha$ Tyr190 and $\alpha$ Asp200, with first-order coupling free energies $\left(\Delta \Delta G_{\text {int }}\right)$ of $-2.47 \mathrm{kcal} / \mathrm{mol}$ (Figure $7 \mathrm{C}$, top face) and $-2.53 \mathrm{cal} /$ mol (Figure $7 \mathrm{C}$, top face), respectively (Table 3 ). Thus, the contribution of Val188 to channel gating depends strongly on both $\alpha$ Tyr 190 and $\alpha$ Asp 200 . Because Val188 directly contacts $\alpha$ Tyr 190 and $\alpha$ Asp200 (17), the interdependence of the residues likely originates from direct inter-residue contact.

To determine whether a third residue affects coupling of 2 residues, we compared the coupling free energies for the 2 residues with and without a third mutated residue. Given that both $\alpha$ Tyr 190 and $\alpha$ Asp200 couple to $\alpha$ Lys 145 (20), we asked whether mutation of Lys 145 affects the strong coupling of Val188 with either of these 2 residues. The analyses indicated that the $\alpha \mathrm{K} 145 \mathrm{E}$ mutation weakened coupling between $\alpha$ Val188 and $\alpha$ Tyr 190, with $\Delta \Delta G_{\text {int }}$ decreasing from -2.47 (Figure 7 C, top face) to $-1.56 \mathrm{kcal} /$ mol (Figure 7C, bottom face). Furthermore, the $\alpha$ K145E mutation also weakened coupling between $\alpha$ Val188 and $\alpha$ Asp200, with $\Delta \Delta G_{\text {int }}$ decreasing from -2.53 (Figure $7 \mathrm{C}$, top face) to $-0.97 \mathrm{kcal} /$ mol (Figure 7C, bottom face). Thus, the magnitude of energetic coupling between the pairs $\alpha \mathrm{V} 188 / \alpha \mathrm{Y} 190$ and $\alpha \mathrm{V} 188 / \alpha \mathrm{D} 200$ depends on $\alpha$ Lys 145 .

Conversely, we asked whether energetic coupling of $\alpha$ Lys 145 with $\alpha$ Tyr 190 and $\alpha$ Asp 200 depends on $\alpha$ Val188. The analyses indicate that coupling between $\alpha$ Lys 145 and $\alpha$ Tyr 190 was weakened by $\alpha V 188 \mathrm{M}$, with $\Delta \Delta G_{\text {int }}$ decreasing from -1.43 without $\alpha \mathrm{V} 188 \mathrm{M}$ (Figure $7 \mathrm{~B}$, left face, and Table 3 ) to $-0.52 \mathrm{kcal} /$ $\mathrm{mol}$ in the presence of $\alpha \mathrm{V} 188 \mathrm{M}$ (Figure 7B, right face). Furthermore, the $\alpha V 188 \mathrm{M}$ mutation also weakened coupling between $\alpha$ Lys 145 and $\alpha$ Asp200, with $\Delta \Delta G_{\text {int }}$ decreasing from -1.66 without $\alpha$ V188M (Figure $7 C$, left face, and Table 3 ) to $-0.10 \mathrm{kcal} /$ $\mathrm{mol}$ in the presence of $\alpha \mathrm{V} 188 \mathrm{M}$ (Figure $7 \mathrm{C}$, right face). Thus, energetic coupling between the pairs $\alpha \mathrm{K} 145 / \alpha \mathrm{Y} 190$ and $\alpha \mathrm{K} 145 /$ $\alpha \mathrm{D} 200$ depends on $\alpha$ Val188.

Within the crystal structure of the $\alpha 1$ extracellular domain, $\alpha$ Tyr 190, $\alpha$ Val188, and $\alpha$ Asp200 form a contiguous chain, but $\alpha$ Val188 does not directly contact $\alpha$ Lys 145 (Figure 7A). Thus, we assessed energetic coupling between $\alpha$ Val188 and $\alpha$ Lys145. Ener- nist-occupied chimeric $\alpha 7$ ligand-binding domain provided evidence for a $\pi$-cation interaction between residues equivalent to $\alpha$ Tyr93 and $\alpha$ Lys 145 in the $\alpha 1$ subunit (30). We therefore tested whether $\alpha$ Val188 is energetically coupled with $\alpha$ Tyr93 in human $\alpha 1$ AChR. The 2-dimensional mutant cycle in Figure 7D shows that $\alpha$ Val188 and $\alpha$ Tyr93 were not energetically coupled (Table 3 ). Thus, although mutation of either residue alone markedly alters channel gating, the combined mutations produce additive changes and thus $\alpha$ Val188 and $\alpha$ Tyr93 are independent of each other. The lack of inter-residue coupling again correlates with the structural data showing that $\alpha$ Tyr93 and $\alpha$ Val188 are spatially separate (Figure 7A).

\section{Discussion}

We identify 2 heteroallelic mutations in the AChR $\alpha 1$ subunit in a patient with severe myasthenic symptoms since birth. The first mutation, $\alpha \mathrm{G} 73 \mathrm{C}$, markedly reduces surface expression of AChR in HEK cells. The second mutation, $\alpha \mathrm{V} 188 \mathrm{M}$, allows robust AChR expression in HEK cells but has a profound effect on receptor activation. Thus, $\alpha \mathrm{V} 188 \mathrm{M}$ determines the phenotype. The functional role of $\alpha$ Val188 has not been investigated in previous mutagenesis studies. To our knowledge, $\alpha V 188 \mathrm{M}$ is the first naturally occurring mutation in the C-loop in the Cys-loop receptor superfamily.

$\alpha \mathrm{V} 188 \mathrm{M}$ decreases gating efficiency approximately 70 -fold and decreases the receptor's affinity for ACh approximately 2 -fold. The fitted rate constants underlying activation of $\alpha \mathrm{V} 188 \mathrm{M} A C h R$ are validated by close correspondence of the predicted burst duration with that recorded with $1 \mu \mathrm{M}$ ACh for the mutant receptor and also by agreement between the predicted $P_{\text {open }}$ and that derived from direct recordings (Figure 6). The findings indicate a compromised safety margin of neuromuscular transmission owing to a reduced $P_{\text {open }}$, an abnormally fast decay of the synaptic current, and a decreased amplitude of synaptic response.

The consequences of $\alpha \mathrm{V} 188 \mathrm{M}$ cannot be attributed to the larger bulk of Met than Val because substitutions with smaller (Gly, Ala, and Thr) or larger (Leu) residues all reduced gating efficiency. However, that Leu and Thr, which like Val have branched side-chains, reduce gating efficiency to a lesser extent than Gly and Ala, suggests that a branched side-chain residue at position $\alpha 188$ is important for the initial transduction of agonist binding to channel gating.

MCA showed that the functional consequences of the mutation $\alpha$ V188M are altered if either $\alpha$ Tyr 190 or $\alpha$ Asp200 is mutated 
A
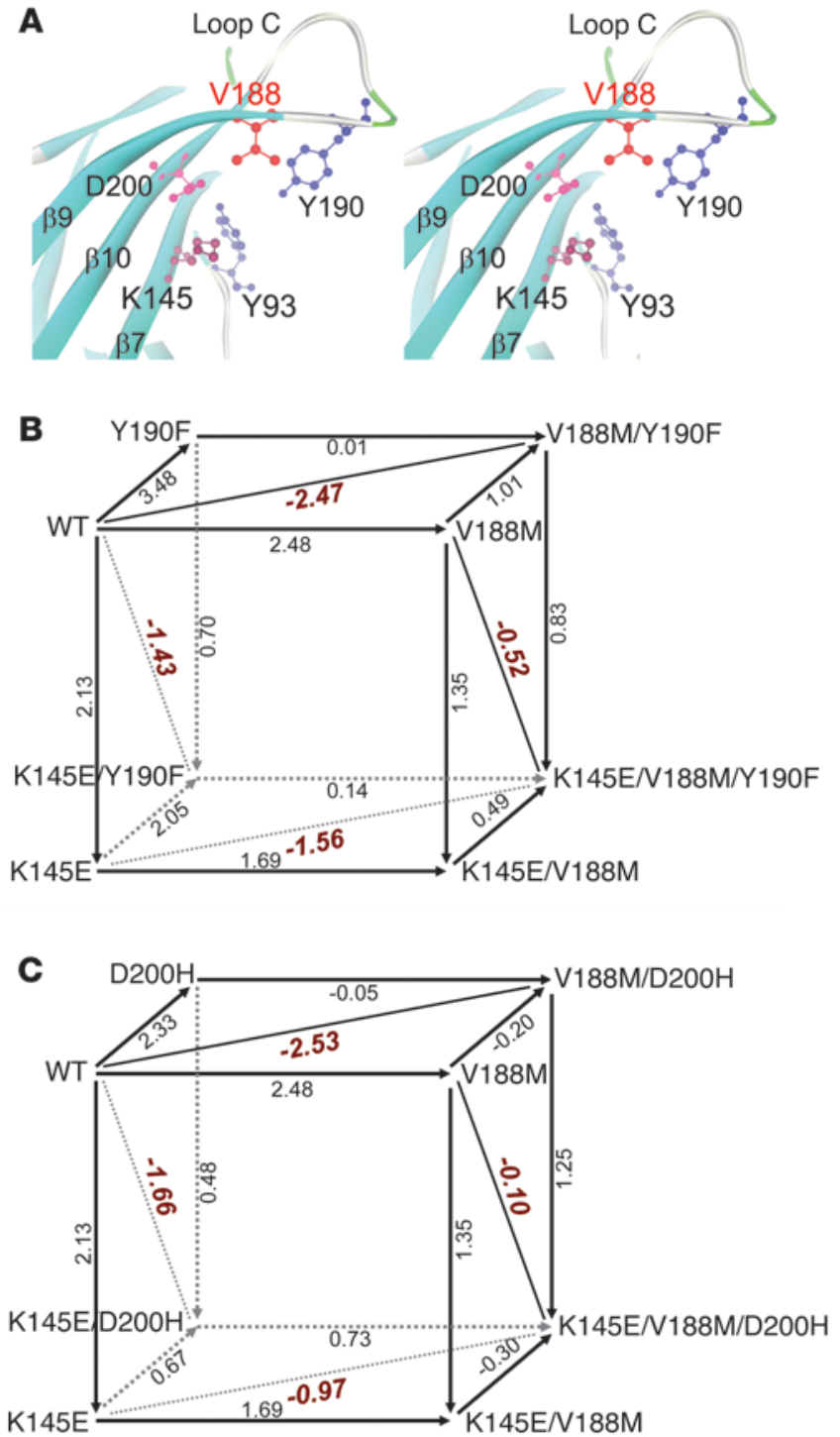

D

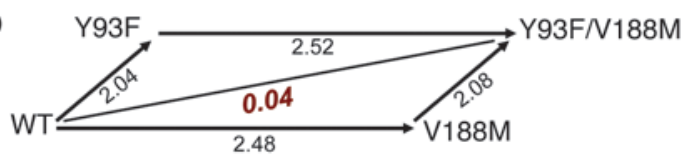

simultaneously. Pairing $\alpha$ V188M with mutants of either $\alpha$ Tyr 190 or $\alpha$ Asp200 revealed large coupling free energies, around -2.5 $\mathrm{kcal} / \mathrm{mol}$, which is similar to the originally reported inter-residue coupling free energy for the pairs $\alpha \mathrm{D} 200 / \alpha \mathrm{K} 145$ and $\alpha \mathrm{Y} 190 /$ $\alpha K 145$ (20). Analysis of the crystal structure of the $\alpha 1$ extracellular domain revealed that $\alpha$ Tyr $190, \alpha$ Val188, and $\alpha$ Asp 200 form a contiguous chain, so their mutual interdependence likely originates from direct interactions.

The crystal structure of the $\alpha 1$ extracellular domain is a reasonable model for the conformation of the resting state of AChR in the region encompassing $\alpha V 188$. This is likely because the $\alpha 1$ structure was obtained with bound $\alpha$-bgt, and the local region is similar to that of acetylcholine-binding protein without bound agonist. In particular, in the $\alpha 1$ structure, $\alpha \operatorname{Tyr} 190$ on the C-loop is far

\section{Figure 7}

The $\alpha 1$ AChR subunit binding site and MCA of potentially interacting residues. (A) Stereo view of the binding site at $1.9-\AA$ resolution (PDB 2QC1) shows spatial disposition of the potentially interacting residues. (B) Cubic mutant cycle of energetic interactions between $\alpha \mathrm{V} 188, \alpha \mathrm{Y} 190$, and $\alpha \mathrm{K} 145$. (C) Cubic MCA of energetic interactions among $\alpha \mathrm{V} 188, \alpha \mathrm{D} 200$, and $\alpha \mathrm{K} 145$. (D) Two-dimensional mutant cycle of energetic interaction among $\alpha \mathrm{V} 188$ and $\alpha \mathrm{Y} 93$. In B-D, numbers on arrows show difference in free energy change $(\Delta \Delta G)$ between 2 different $A C h R s$ in $\mathrm{kcal} / \mathrm{mol}$. The indicated diagonal lines in planes show first-order coupling free energy $\left(\Delta \Delta G_{\text {int }}\right)$ for a given pair of mutants. For the front planes in $\mathbf{B}$ and $\mathbf{C}, \Delta \Delta G_{\text {int }}=-0.78 \mathrm{kcal} / \mathrm{mol}$; for the back planes in $\mathbf{B}$ and $\mathbf{C}, \Delta \Delta G_{\text {int }}=0.13$ and $0.78 \mathrm{kcal} / \mathrm{mol}$, respectively.

from $\alpha$ Lys145 on $\beta$-strand 7. In the available structures with agonist bound, the C-loop adopts a closed conformation that brings $\alpha$ Tyr190 into close register with Lys 145 , forming a strong hydrogen bond $(18,19,29,30)$. Furthermore, in the crystal structure of an $\alpha 7$ chimeric ligand-binding domain with bound agonist, an Arg residue at the position equivalent to $\alpha$ Val188 establishes close register with the residue equivalent to $\alpha \operatorname{Tyr} 190$ through a $\pi$-cation interaction (30). Combined with these structural data, our findings suggest that $\alpha$ Val188, through an ideal steric fit, enables the proper interaction of $\alpha$ Tyr 190 with $\alpha$ Lys 145 . Furthermore, in both the resting and agonist-bound conformations, the steric properties of $\alpha$ Val188 enable formation of a stable salt bridge between $\alpha$ Asp200 and $\alpha$ Lys 145 . Thus, in the context of the available crystallography and mutagenesis data, our functional studies indicate that $\alpha$ Val188 is a key residue within the network of residues that initiates coupling of agonist binding to channel gating.

$\alpha$ Val188 is not conserved among subtypes of nicotinic AChR $\alpha$ subunits, with neuronal AChRs containing Ile, Leu, Arg, or Lys at equivalent positions. Our findings show that $\alpha$ Val188 is an essential contributor to the triad of conserved residues that initiate coupling of agonist binding to channel gating, suggesting that among different types of nicotinic AChRs, sequence variations at positions equivalent to $\alpha$ Val18 enable tuning of the synaptic response according to physiological needs.

\section{Methods}

Mutation analysis. We directly sequenced $\mathrm{ACh} R \alpha 1, \beta 1, \delta$, and $\varepsilon$ subunit genes using genomic DNA. The mutations were traced with allele-specific PCR in family members and in 400 normal alleles of 200 unrelated controls.

Construction and expression of wild-type and mutant AChRs. Sources of human $\alpha, \beta, \varepsilon$, and $\delta$ subunit cDNAs were as previously described (32). All 4 cDNAs were subcloned into the CMV-based expression vector pRBG4 for expression in HEK293 cells (32). The artificial mutations were engineered into wild-type AChR subunit cDNAs in PRBG4 using the QuikChange Site-Directed Mutagenesis Kit (Stratagene). The presence of each mutation and absence of unwanted mutations were confirmed by sequencing the entire inserts. HEK cells were transfected with plasmids comprising pRBG4- $\alpha,-\beta,-\varepsilon$, and $-\delta$ and pEGFP-N1 at a ratio of $2: 1: 1: 1: 1$, using FuGENE 6 transfection reagent (Roche).

$\alpha$-bgt binding measurements. The total number of $\left[{ }^{125} \mathrm{I}\right] \alpha$-bgt sites on the surface of transfected HEK cells was determined as previously described (32).

Patch-clamp recordings and single-channel kinetic analysis. Recordings were obtained in the cell-attached configuration at a membrane potential of -80 $\mathrm{mV}$ at $22^{\circ} \mathrm{C}$ and with bath and pipette solutions containing (in $\mathrm{mM}$ ): $\mathrm{KCl}$, 142; $\mathrm{NaCl}, 5.4 ; \mathrm{CaCl}_{2}, 1.8 ; \mathrm{MgCl}_{2}, 1.7 ; \mathrm{HEPES}, 10$, pH 7.4. Single-channel currents were recorded using an Axopatch 200A amplifier (Axon Instru- 
ments) at a bandwidth of $50 \mathrm{kHz}$, digitized at 5 - $\mu$ s intervals using a Digidata $1322 \mathrm{~A}$ (Axon Instruments) and recorded to hard disk using the program Clampex 8.2 (Axon Instruments). Recordings obtained with ACh at $1 \mu \mathrm{M}$ or less were analyzed at a uniform bandwidth of $11.7 \mathrm{kHz}$ with dead time of $15.3 \mu$ s imposed. Recordings obtained with ACh at $10 \mu \mathrm{M}$ or more were analyzed with dead time at $25 \mu$ s at $10 \mathrm{kHz}$ with TACx4.0.9 software (Bruxton). Dwell-time histograms were plotted on a logarithmic abscissa and fitted by the sum of exponentials by maximum likelihood (33).

To estimate rate constants underlying AChR activation, we employed desensitizing concentrations of ACh that cause events from a single channel to cluster into identifiable activation episodes (34). Clusters were identified as a series of closely spaced openings preceded and followed by closed intervals greater than a defined critical time. The critical time was determined by a method that misclassifies an equal number of events between 2 adjacent closed-time components (35). For each receptor, the critical time that provided the best fit for the closed-time histogram was chosen for the final analysis. Clusters with fewer than 5 openings were excluded from analysis. Individual clusters were examined for homogeneity by determination of the mean $P_{o p e n}$ and open duration for each cluster, and clusters within 2 SDs of the mean were accepted for further analysis $(36,37)$. The resulting global set of open and closed dwell times of wild-type and mutant AChRs were analyzed using the program MIL (QuB suite; http://www.qub.buffalo.edu/wiki/index.php/Main_Page), which uses an interval-based maximum likelihood method that also corrects for missed events (38). The kinetic analysis of wild-type and mutant receptors yielded a set of rate constants that were fitted to a scheme of receptor activation shown in Figure 5.

For each type of AChR, single-channel dwell times obtained over a range of ACh concentrations were fitted simultaneously. Data were obtained over a range of $\mathrm{ACh}$ concentrations from 10 to $1,000 \mu \mathrm{M}$ for wild-type, $\alpha \mathrm{V} 188 \mathrm{~A}$, and $\alpha \mathrm{K} 145 \mathrm{E}$ AChRs; from 30 to $1,000 \mu \mathrm{M}$ for $\alpha \mathrm{V} 188 \mathrm{M}, \alpha \mathrm{V} 188 \mathrm{G}$, $\alpha V 188 \mathrm{~T}, \alpha \mathrm{V} 188 \mathrm{~L}$, and $\alpha \mathrm{D} 200 \mathrm{H}$ AChRs; from 100 to $1,000 \mu \mathrm{M}$ for $\alpha \mathrm{Y} 93 \mathrm{~F}$ and $\alpha \mathrm{K} 145 \mathrm{E} / \alpha \mathrm{D} 200 \mathrm{H}$ AChRs; and 1,000 $\mu \mathrm{M}$ for others. The final set of rate constants was checked by superimposing probability density functions calculated from the rate constants on the experimental dwell time histograms and by the ability of the rate constants to predict burst length at low ACh concentrations and the $P_{\text {open }}(39,40)$.

$M C A$. We used MCA to determine whether the functional contributions of 2 residues in the AChR, $\mathrm{X}$ and $\mathrm{Y}$, are interdependent. The analysis depends on examining the functional consequences of mutating residue $\mathrm{X}$ without and with mutation of residue $\mathrm{Y}$ and quantifying the free energy change associated with a given state change for the mutants $A C h R_{X}, A C h R_{Y}$, and $A C h R_{X Y}$. In the case of the AChR, the relevant state change was defined by the channel gating equilibrium constant of the di-liganded receptor, $\theta$, which has an associated free energy change $\Delta G=-R T \ln \theta$. Although recent studies show that $\theta$ is a composite of closedstate priming and channel gating steps (28) and thus is an apparent equilibrium constant, it still remains a highly sensitive measure of inter-residue coupling. The changes in gating free energy due to the mutations $\mathrm{X}, \mathrm{Y}$, and $X Y$ relative to the wild-type are designated $\Delta \Delta G_{X}, \Delta \Delta G_{Y}$, and $\Delta \Delta G_{X Y}$. These terms are related to the free energy of inter-residue interaction, $\Delta \Delta G_{i n t}$, as follows: $\Delta \Delta G_{X Y}=\Delta \Delta G_{X}+\Delta \Delta G_{Y}+\Delta \Delta G_{\text {int }}(31,41,42)$. Given this relationship and noting that $\Delta \Delta G$ s for the individual mutant AChR is $\Delta G_{X}-\Delta G_{W}, \Delta G_{Y}-$ $\Delta G_{W}, \Delta G_{X Y}-\Delta G_{W}, \Delta \Delta G_{\text {int }}$ can be calculated from $-R T \ln \left[\left(\theta_{W} \times \theta_{X Y}\right) /\left(\theta_{X} \times \theta_{Y}\right)\right]$, where $\theta$ is the measured channel gating equilibrium constant for wild-type (W) and mutant $(X, Y, X Y)$ AChRs. To determine interactions between 3 sets of residues, we used cubic MCA, where the faces of the cube are derived from 2-dimensional mutant cycles.

Statistics. Wild-type and mutant values in Table 1 were compared by the 2 -tailed Student's $t$ test. Standard error estimates of activation rate constant of wild-type and mutant receptors shown in Tables 2 and 3 were computed by the MIL program from the curvature of the likelihood function at its maximum (36). A $P$ value less than 0.05 was considered significant.

Study approval. All human studies were in accordance with the guidelines of, and approved by, the Institutional Review Board of the Mayo Clinic. The patient reported in this study provided informed consent to participate in the study.

\section{Acknowledgments}

This work was supported by NIH grants to A.G. Engel (NS-6277) and to S.M. Sine (NS-31744) and by a Muscular Dystrophy Association Grant to A.G. Engel.

Received for publication February 16, 2012, and accepted in revised form April 18, 2012.

Address correspondence to: Xin-Ming Shen, Department of Neurology, Mayo Clinic, 200 First Street SW, Rochester, Minnesota 55905, USA. Phone: 507.284.5102; Fax: 507.284.5831; E-mail: shen.xinming@mayo.edu.
1. Engel AG. Current status of the congenital myasthenic syndromes. Neuromuscul Disord. 2012;22(2):99-111.

2. Engel AG, Ohno K, Sine SM. Sleuthing molecular targets for neurological diseases at the neuromuscular junction. Nat Rev Neurosci. 2003;4(5):339-352.

3. Ohno K, et al. Choline acetyltransferase mutations cause myasthenic syndrome associated with episodic apnea in humans. Proc Natl Acad Sci U S A. 2001;98(4):2017-2022.

4. Ohno K, Brengman JM, Tsujino A, Engel AG. Human endplate acetylcholinesterase deficiency caused by mutations in the collagen-like tail subunit (ColQ) of the asymmetric enzyme. Proc Natl Acad Sci U S A. 1998;95(16):9654-9659.

5 . Donger $\mathrm{C}$, et al. Mutation in the human acetylcholinesterase-associated gene, $C O L Q$, is responsible for congenital myasthenic syndrome with endplate acetylcholinesterase deficiency. Am J Hum Genet. 1998;63(4):967-975.

6. Ohno K, et al. Rapsyn mutations in humans cause endplate acetylcholine receptor deficiency and myasthenic syndrome. Am J Hum Genet. 2002; $70(4): 875-885$.

7. Huze $\mathrm{C}$, et al. Identification of an agrin mutation that causes congenital myasthenia and affects syn- apse function. Am J Hum Genet. 2009;85(2):155-167. 8. Chevessier F, et al. MUSK, a new target for mutations causing congenital myasthenic syndrome. Hum Mol Genet. 2004;13(24):3229-3240.

9. Beeson D, et al. Dok-7 mutations underlie a neuromuscular junction synaptopathy. Science. 2006; 313(5795):1975-1978.

10. Selcen D, et al. Myasthenic syndrome caused by plectinopathy. Neurology. 2011;76(4):327-336.

11. Senderek J, et al. Hexosamine biosynthetic pathway mutations cause neuromuscular transmission defect. Am J Hum Genet. 2011;88(2):162-172.

12. Tsujino A, et al. Myasthenic syndrome caused by mutation of the SCN 4 A sodium channel. Proc Natl Acad Sci U S A. 2003;100(12):7377-7382.

13. Sine SM, Engel AG. Recent advances in Cys-loop receptor structure and function. Nature. 2006 440(7083):448-455.

14. Galzi JL, Revah F, Bessis A, Changeux JP. Functional architecture of the nicotinic acetylcholine receptor: from electric organ to brain. Annu Rev Pharmacol Toxicol. 1991;31:37-72.

15. Unwin N. Refined structure of the nicotinic acetylcholine receptor at 4 Å resolution. J Mol Biol. 2005; 346(4):967-989.
16. Sine SM. The nicotinic receptor ligand binding domain. J Neurobiol. 2002;53(4):431-446.

17. Dellisanti CD, Yao Y, Stroud JC, Wang ZZ, Chen L. Crystal structure of the extracellular domain of the nAChR $\alpha 1$ bound to $\alpha$-bungarotoxin at $1.94 \AA$ resolution. Nat Neurosci. 2007;10(8):953-962.

18. Brejc K, van Dijk WV, Schuurmans M, van der Oost J, Smit AB, Sixma TK. Crystal structure of ACh-binding protein reveals the ligand-binding domain of nicotinic receptors. Nature. 2001;411(6835):269-276.

19. Hansen SB, Sulzenbacher G, Huxford T, Marchot P, Taylor P, Bourne Y. Structures of Aplysia AChBP complexes with nicotinic agonists and antagonists reveal distinctive binding interfaces and conformations. EMBO J. 2005;24(20):3635-3646.

20. Mukhtasimova N, Free C, Sine SM. Initial coupling of binding to gating mediated by conserved residues in muscle nicotinic receptor. J Gen Physiol. 2005;126(1):23-39.

21. Lee WY, Sine SM. Principal pathway coupling agonist binding to channel gating in nicotinic receptors. Nature. 2005;438(7065):243-247.

22. Shen XM, Fukuda T, Ohno K, Sine SM, Engel AG. Congenital myasthenia-related AChR $\delta$ subunit mutation interferes with intersubunit communica- 
tion essential for channel gating. J Clin Invest. 2008; 118(5):1867-1876.

23. Lee WY, Free CR, Sine SM. Nicotinic receptor interloop proline anchors $\beta 1-\beta 2$ and Cys loops in coupling agonist binding to channel gating.J Gen Physiol. 2008;132(2):265-278.

24. Wang HL, et al. Single-channel current through nicotinic receptor produced by closure of binding C-loop. Biophys J. 2009;96(9):3582-3590.

25. Gao F, et al. Agonist mediated conformational changes in acetylcholine-binding protein revealed by simulation and intrinsic tryptophan fluorescence. J Biol Chem. 2005;280(9):8443-8451.

26. Cheng X, Wang H, Grant B, Sine SM, McCammon A. Targeted molecular dynamics study of C-loop closure and channel gating in nicotinic receptors. PLoS Comput Biol. 2006;2(9):e134.

27. Lee WY, Free CR, Sine SM. Binding to gating transduction in nicotinic receptors: Cys-loop energetically couples to pre-M1 and M2-M3 regions. J Neurosci. 2009;29(10):3189-3199.

28. Mukhtasimova N, Lee WY, Wang HL, Sine SM. Detection and trapping of intermediate states priming nicotinic receptor channel opening. Nature. 2009;459(7245):451-454.

29. Celie PH, van Rossum-Fikkert SE, van Dijk WJ,
Brejc K, Smit AB, Sixma TK. Nicotine and carbamycholine binding to nicotinic acetylcholine receptors as studied in AChBP crystal structures. Neuron. 2004;41(6):907-914.

30. Li SX, et al. Ligand-binding domain of an $\alpha 7$ nicotinic receptor chimera and its complex with agonist. Nat Neurosci. 2011;14(10):1253-1259.

31. Horovitz A, Fersht A. Strategy for analyzing the cooperativity of intramolecular interactions in peptides and proteins. J Mol Biol. 1990;214(3):613-617.

32. Ohno K, et al. Congenital myasthenic syndrome caused by decreased agonist binding affinity due to a mutation in the acetylcholine receptor $\delta$ subunit. Neuron. 1996;17(1):157-170.

33. Sigworth FJ, Sine SM. Data transformation for improved display and fitting of single-channel dwell time histograms. Biophys J. 1987;52(6):1047-1054.

34. Sakmann B, Patlak J, Neher E. Single acetylcholine-activated channels show burst kinetics in the presence of desensitizing concentrations of agonist. Nature. 1980;286(5768):71-73.

35. Colquhoun D, Sakmann B. Fast events in single channel currents activated by acetylcholine and its analogues at the frog muscle end-plate. J Physiol. 1985;369:501-557.

36. Qin F, Auerbach A, Sachs F. Maximum likelihood estimation of aggregated Markov process. Pro Biol Sci. 1997;264(1380):375-383.

37. Shen XM, et al. Mutation causing severe myasthenia reveals functional asymmetry of AChR signature Cys-loops in agonist binding and gating. JClin Invest. 2003;111(4):497-505.

38. Qin F, Auerbach A, Sachs F. Estimating singlechannel kinetic parameters from idealized patchclamp data containing missed events. Biophys J. 1996;70(1):264-280.

39. Colquhoun D, Hawkes AG. The principles of the stochastic interpretation of ion channel mechanisms. In: Sakmann B, Neher E, eds. Single-channel Recording. New York, New York, USA: Plenum Press; 1995:397-482.

40. Colquhoun D, Hawkes AG. A Q-matrix cookbook: how to write only one program to calculate the single-channel and macroscopic predictions for any kinetic mechanism. In: Sakmann B, Neher E, eds. Single-channel Recording. New York, New York, USA: Plenum Press; 1995:589-633.

41. Wells JA. Additivity of mutational effects in proteins. Biochemistry. 1990;29(37):8509-8517.

42. Horovitz A. Double-mutant cycles: a powerful tool for analyzing protein structure and function. Fold Des. 1996;1(6):R121-R126. 